\title{
Macrophage hypoxia signaling regulates cardiac fibrosis via Oncostatin M
}

\author{
Hajime Abe ${ }^{1,2}$, Norihiko Takeda1,3, Takayuki Isagawa4, Hiroaki Semba1,5, Satoshi Nishimura ${ }^{3,6}$,

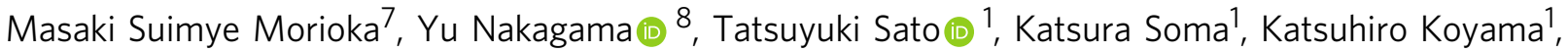 \\ Masaki Wake ${ }^{1}$, Manami Katoh ${ }^{1}$, Masataka Asagiri ${ }^{9}$, Michael L. Neugent ${ }^{10}$, Jung-whan Kim $^{10}$, \\ Christian Stockmann ${ }^{11,12}$, Tomo Yonezawa ${ }^{13}$, Ryo Inuzuka ${ }^{8}$, Yasushi Hirota (1) ${ }^{14}$, Koji Maemura ${ }^{4}$ \\ Takeshi Yamashita (D) ${ }^{5}$, Kinya Otsu ${ }^{2}$, Ichiro Manabe ${ }^{15}$, Ryozo Nagai ${ }^{16}$ \& Issei Komuro ${ }^{1}$
}

The fibrogenic response in tissue-resident fibroblasts is determined by the balance between activation and repression signals from the tissue microenvironment. While the molecular pathways by which transforming growth factor- 1 (TGF- $\beta 1$ ) activates pro-fibrogenic mechanisms have been extensively studied and are recognized critical during fibrosis development, the factors regulating TGF- $\beta 1$ signaling are poorly understood. Here we show that macrophage hypoxia signaling suppresses excessive fibrosis in a heart via oncostatin-m (OSM) secretion. During cardiac remodeling, Ly6Chi monocytes/macrophages accumulate in hypoxic areas through a hypoxia-inducible factor (HIF)-1 $\alpha$ dependent manner and suppresses cardiac fibroblast activation. As an underlying molecular mechanism, we identify OSM, part of the interleukin 6 cytokine family, as a HIF-1 $\alpha$ target gene, which directly inhibits the TGF- $\beta 1$ mediated activation of cardiac fibroblasts through extracellular signal-regulated kinase 1/2dependent phosphorylation of the SMAD linker region. These results demonstrate that macrophage hypoxia signaling regulates fibroblast activation through OSM secretion in vivo.

\footnotetext{
${ }^{1}$ Department of Cardiovascular Medicine, Graduate School of Medicine, The University of Tokyo, 7-3-1 Hongo, Bunkyo-ku, Tokyo 113-8655, Japan. ${ }^{2}$ The School of Cardiovascular Medicine and Sciences, King's College London British Heart Foundation Centre of Excellence, London SE5 9NU, UK. ${ }^{3}$ PRESTO, JST, 4-1-8 Honcho Kawaguchi, Saitama 332-0012, Japan. ${ }^{4}$ Graduate School of Biomedical Science, Nagasaki University, 1-7-1sakamoto, Nagasaki 8528501, Japan. ${ }^{5}$ Department of Cardiovascular Medicine, The Cardiovascular Institute, 3-2-19 Nishiazabu, Minato-ku, Tokyo 106-00031, Japan. ${ }^{6}$ Center for Molecular Medicine, Jichi Medical University, 3311-1 Yakushiji, Shimotsuke-shi, Tochigi 329-0498, Japan. ${ }^{7}$ Depertment of Bioinformatics, Medical Research Institute, Tokyo Medical and Dental University, 1-5-45 Yushima, Bunkyoku, Tokyo 113-8510, Japan. ${ }^{8}$ Department of Pediatrics, Graduate School of Medicine, The University of Tokyo, 7-3-1 Hongo, Bunkyo-ku, Tokyo 113-8655, Japan. ${ }^{9}$ Department of Pathobiology, Graduate School of Pharmaceutical Sciences, Nagoya City University, 3-1 Tanabe-dori, Mizuho-ku, Nagoya 467-8603, Japan. ${ }^{10}$ Department of Biological Sciences, The University of Texas at Dallas, 800W. Campbell Road FO 3.704G, Richardson, TX 75080, USA. ${ }^{11}$ Institute of Anatomy, University of Zurich, Zurich CH-8057, Switzerland.

${ }^{12}$ Cancer Research Center Zurich, Winterthurerstrasse 190, CH-8057 Zurich, Switzerland. ${ }^{13}$ Center for Therapeutic Innovation, Gene Research Center, Center for Frontier Life Sciences, Nagasaki University, Graduate School of Biomedical Sciences, 1-12-14 Sakamoto, Nagasaki 852-8523, Japan.

${ }^{14}$ Department of Obstetrics and Gynecology, Graduate School of Medicine, The University of Tokyo, 7-3-1 Hongo, Bunkyo-ku, Tokyo 113-8655, Japan.

15 Department of Disease Biology and Molecular Medicine, Graduate School of Medicine, Chiba University, 1-8-1 Inohana, Chuo-ku, Chiba-shi, Chiba 2608670, Japan. ${ }^{16}$ Jichi Medical University, 3311-1 Yakushiji, Shimotsuke-shi, Tochigi-ken, Tochigi 329-0498, Japan. Correspondence and requests for materials should be addressed to N.T. (email: ntakeda-tky@umin.ac.jp)
} 
C ardiac remodeling occurs after myocardial infarction or pressure overload. During the remodeling processes, activated fibroblasts (myofibroblasts) cause cardiac fibrosis development in the infarcted areas of the myocardium in order to support the structure of the heart ${ }^{1-3}$. Cardiac fibrosis also occurs in pressure overload-induced cardiac remodeling, leading to the development of heart failure with preserved ejection fraction (HFpEF), a form of congestive heart failure in which the fraction of blood ejected from the left ventricle is within normal thresholds $(>50 \%)^{4}$. In both myocardial infarction and pressure overload-induced cardiac fibrosis, the extent of interstitial fibrosis correlates with mortality and major cardiovascular adverse event rates in heart failure patients ${ }^{5-8}$. Therefore, elucidation of the molecular processes by which fibroblasts are activated or deactivated is critically important for the development of therapeutic approaches in the management of cardiac fibrosis.

During fibrogenesis, several subsets of monocytes/macrophages $(\mathrm{M} \Phi)$ are recruited to the heart including both pro-inflammatory $\left(\right.$ Ly6 $\mathrm{C}^{\mathrm{hi}}$ ) and anti-inflammatory $\left(\mathrm{Ly} 6 \mathrm{C}^{\mathrm{lo}}\right) \mathrm{M} \Phi^{9}$ and play a critical role in tissue remodeling. The extent of inflammation significantly correlates to the severity of heart failure ${ }^{10-12}$. Notably, local oxygen concentration within inflamed areas tends to be decreased, leading to tissue hypoxia ${ }^{13,14}$. The transcription factor, hypoxia-inducible factor (HIF)- $1 \alpha$ and HIF-2 $\alpha$ are stabilized under hypoxic conditions. In its stable form, HIF-as induce target gene expression through their binding to the hypoxia response elements (HREs) of target genes ${ }^{15-23}$. We previously showed that HIF- $1 \alpha$ and HIF- $2 \alpha$ are highly expressed in pro-inflammatory and anti-inflammatory $M \Phi$, respectively ${ }^{24}$. During cell migration, cytosolic ATP is rapidly consumed at the filopodia or lamellipodia. Recently, we revealed that HIF-1a mediated glycolytic reprogramming plays a key role for $M \Phi$ to mobilize towards the hypoxic tissue ${ }^{25}$. Pyruvate kinase, muscle type, a glycolytic enzyme capable of producing ATP, localizes in the filopodia and lamellipodia, which may account for the beneficial roles of glycolytic metabolism in $М \Phi$ migration.

The roles of $M \Phi$, however, in tissue fibrosis have not been fully elucidated $^{9,26}$. The current study examine the roles of $M \Phi$ in cardiac fibrosis using murine model of cardiac remodeling. The results demonstrate that $\mathrm{Ly} 6 \mathrm{C}^{\text {hi }} \mathrm{M} \Phi$ accumulate in hypoxic areas and suppress excessive fibrosis in cardiac tissue by secreting a cytokine Oncostatin-M (OSM) in vivo.

\section{Results}

Ly6Chi/lo $M \Phi$ accumulate to the heart after TAC procedure. TAC is a commonly used experimental model for inducing cardiac fibrosis. To identify the population of cardiac inflammatory cells which accumulates in the heart tissue, we performed fluorescence-activated cell sorting (FACS) analysis of cardiac tissues after administering the TAC operation. In this study, we defined cardiac $\mathrm{M} \Phi$ as $\mathrm{CD}_{11 \mathrm{~b}}{ }^{+}, \mathrm{F} 4 / 80^{+}$, $\mathrm{Ly}_{6 \mathrm{G}}{ }^{-}$mononuclear cells $^{27}$. Cardiac $M \Phi$ acutely, but transiently accumulate in the heart after TAC (Fig. 1a). Ly6C is known as a surface marker for defining cardiac $\mathrm{M} \Phi$ subpopulations ${ }^{28}$. We next isolated two $\mathrm{M} \Phi$ subsets including Ly6Chi $\mathrm{M} \Phi$ and Ly6C ${ }^{\text {lo }} M \Phi$ (Supplementary Fig. 1) ${ }^{9,29}$. Cardiac Ly6C hi $\mathrm{M} \Phi$ highly express Interleukin 1 beta (Illb) and C-C chemokine receptor type 2 (Ccr2), whereas Ly6Clo MФ express Mannose receptor, $C$ type $1(\mathrm{Mrc1})$ and Arginase 1 (Arg1) (Supplementary Fig. 2). Most of the cells recruited at day 3

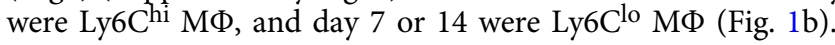

Ly6 $\mathrm{C}^{\text {hi }} \mathrm{M \Phi}$ accumulate in hypoxic regions. Chemokine (C-C motif) ligand $2(\mathrm{Ccl} 2)$ and chemokine (C-X3-C motif) ligand 1 are chemokines, which have a potential to recruit Ly6Chi and Ly6 $C^{\text {lo }} M \Phi$, respectively ${ }^{9}$. While the recruited $M \Phi$ presumably modulates cardiac fibrosis, its migration process is significantly influenced by tissue oxygenation ${ }^{14,25}$. To test whether hypoxic areas develop during cardiac remodeling, we performed in vivo two photon microscopic analyses using a phosphorescent hypoxia probe, LOX-1. Striking augmentation of LOX-1 signal was observed at 3 days after TAC operation, reflecting tissue hypoxia (Fig. 1c). We used another hypoxia probe, pimonidazole (Pimo), to further examine the oxygenation status of cardiac tissues ${ }^{30}$. Pimo staining was visualized in the whole area of the heart at 3 days after TAC (Supplementary Fig. 3). To clarify the molecular link between mechanical stress and tissue hypoxia, we isolated primary cardiomyocytes and measured the oxygen consumption rate (OCR) using flux analyzer (XF24, Agilent Technologies, USA). OCR of the cardiomyocytes was increased at day 3 after TAC operation (Supplementary Fig. 4). These results indicate a hypothesis that elevated oxygen demand in cardiomyocytes underlies the occurrence of tissue hypoxia in pressure overloaded heart.

We next measured Pimo signals in cardiac MФ. Ly6C ${ }^{\text {hi }} M \Phi$ displayed a higher Pimo signal intensity than Ly6Clo $\mathrm{M} \Phi$, suggesting that Ly6C $\mathrm{C}^{\text {hi }} \mathrm{M} \Phi$ indeed accumulate in hypoxic areas in vivo (Fig. 1d). To test the roles of HIF-1a signaling in cardiac $\mathrm{M} \Phi$ distribution, we generated myeloid-specific HIF-1a knockout mice (HIF-1 $\alpha^{\text {flox/flox; }}$ LysM-cre $e^{+/-}$, mHIF-1a CKO $)^{31,32}$. The deletion efficiency of the HIF-1 $\alpha$ gene at the mRNA level was more than $65 \%$ in both Ly6 $\mathrm{C}^{\text {hi }} \mathrm{M} \Phi$ and $\mathrm{Ly} 6 \mathrm{C}^{\text {lo }} \mathrm{M} \Phi$ in the heart (Supplementary Fig. 5). The baseline cardiac function was unaltered in mHIF-1a CKO mice compared with cre negative controls (Supplementary Fig. 6). We next performed the TAC procedure in mHIF-1a CKO mice. The results showed that the cardiac accumulation of $\mathrm{Ly} 6 \mathrm{C}^{\mathrm{hi}} \mathrm{M} \Phi$ at day 3 was strikingly decreased in mHIF-1a CKO, whereas that of Ly6C ${ }^{\text {lo }} \mathrm{M} \Phi$ was not affected (Fig. 1e). These results show that HIF-1a signaling plays an integral role in the recruitment of $\mathrm{Ly} 6 \mathrm{C}^{\text {hi }} \mathrm{M} \Phi$ to the hypoxic area of the heart, but not of Ly6C ${ }^{\text {lo }} \mathrm{M} \Phi$.

Cardiac fibrosis develops in HIF-1a knockout mice. We next measured the fibrotic area and found that it was significantly larger in mHIF-1a CKO mice compared with the controls (Fig. 2a). Whereas the number of CD31-positive cells remained unchanged, the number of alpha-smooth muscle actin (aSMA)positive myofibroblasts was increased in mHIF-1 1 CKO mice (Fig. 2b, Supplementary Fig. 7). The number of TdT-mediated dUTP nick end labeling (TUNEL)-positive cells was not increased in mHIF-1a CKO mice (Supplementary Fig. 8), indicating that loss of myeloid cell-specific HIF-1a did not affect cardiomyocyte survival in vivo. While these results indicate the anti-fibrotic activity of $\operatorname{Ly} 6 \mathrm{C}^{\mathrm{hi}} \mathrm{M} \Phi$, the roles of Ly6 $\mathrm{C}^{\text {lo }} \mathrm{M} \Phi$ in cardiac fibrosis still remained unclear. Therefore, we injected clodronate liposomes intraperitoneally to further deplete $\mathrm{Ly}_{6} \mathrm{C}^{\text {lo }} \mathrm{M} \Phi$ in mHIF-1a CKO mice. Injection of clodronate liposomes did not affect the extent of cardiac fibrosis (Supplementary Fig. 9). These results suggest that Ly6C ${ }^{\text {hi }} \mathrm{M} \Phi$ hypoxia signaling elicits the major role in regulating cardiac fibrosis in vivo. In addition, mHIF-1a CKO mice had significantly decreased left ventricular ejection fractions along with increased heart weights (Fig. 2c, d and Supplementary Figs. 10, 11) as well as markedly decreased survival after the TAC procedure (Fig. 2e). These data indicate that $M \Phi$ hypoxia signaling has a cardio-protective role in TAC model. To further examine the roles of MФ HIF-1a signaling in the development of replacement fibrosis, we performed myocardial infarction model using mHIF-1a CKO mice. Fibrotic area in myocardial infarction model was significantly larger in mHIF-1a CKO mice compared with controls (Supplementary Fig. 12). 

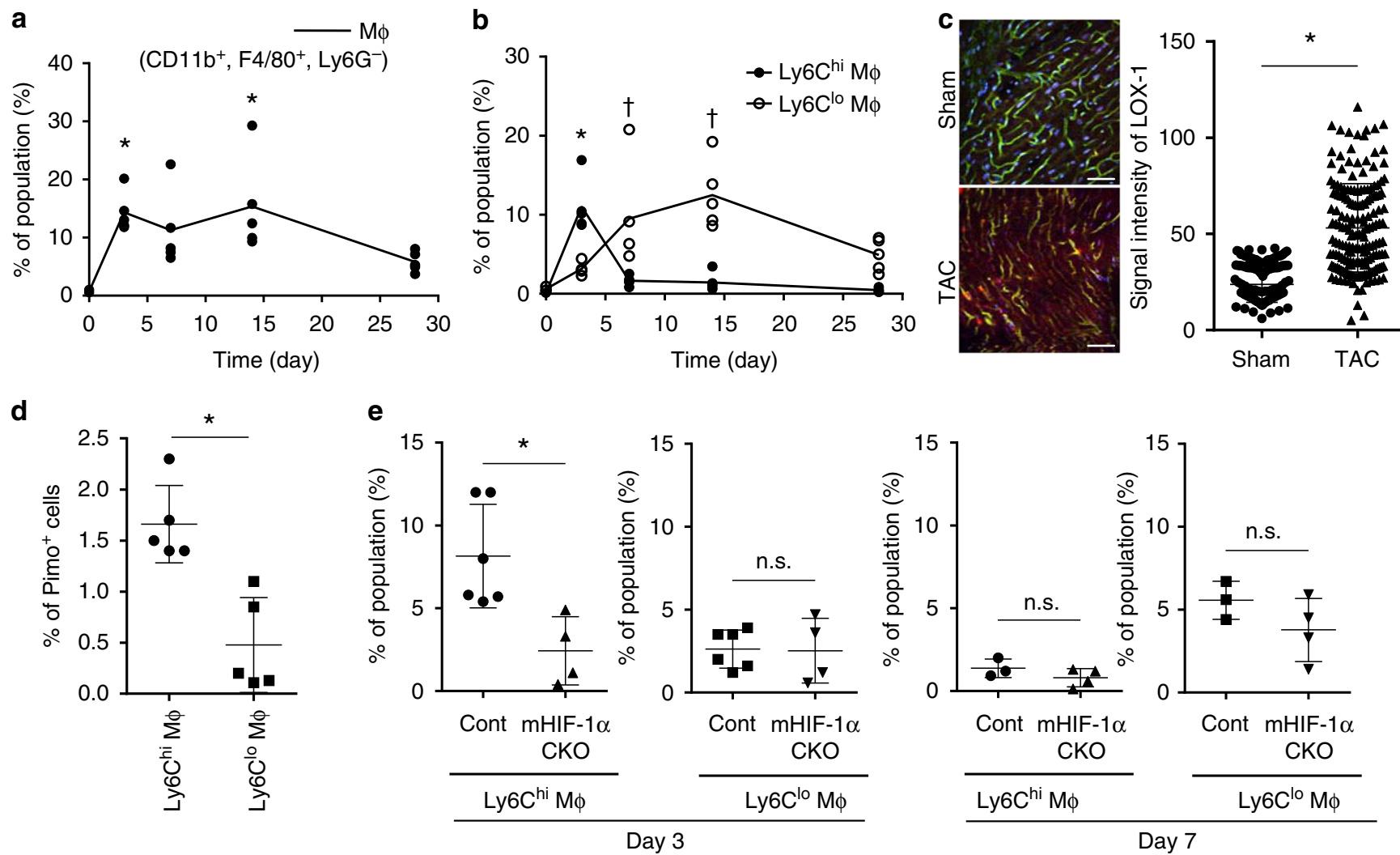

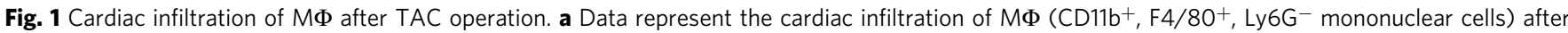
TAC operation. b Data represent the cardiac infiltration of Ly6Chi $M \Phi$ and Ly6Clo $M \Phi$ after TAC operation. ${ }^{*}$ Ly $6 C$ hi $M \Phi$. ' $L y 6 C$ lo $M \Phi$. The Kruskal-Wallis test was used for the statistical analysis. (Figure $\mathbf{a}, \mathbf{b} ; n=5$ at day $0,3,7,14$, and 28). c Hypoxic area was visualized in TAC operated mice ( 3 days after TAC) using LOX-1 (red). Vasculatures and nucleus were labeled with isolectin (green) and Hoechst 33342 (blue) (left). Scale bar $=50 \mu \mathrm{m}$. LOX-1 signal intensity was calculated in sham or TAC operated mouse (right). (ROI number 180, sham and TAC operated mouse, respectively). d M $\Phi$ after TAC operation were stained with Pimonidazole (Pimo). The population of Pimo positive cells in Ly6Chi M $\Phi$ (day $3, n=5)$ and Ly6Clo M $\Phi($ day $7, n=5$ ) are

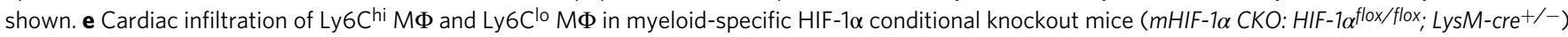
were analyzed 3 or 7 days after TAC operation. Cre negative littermates were used as a control (cont). Day 3, cont $(n=6)$ and mHIF-1 $\alpha$ CKO $(n=4)$. Day 7, cont $(n=3)$ and mHIF-1 $\alpha$ CKO $(n=4)$. The Mann-Whitney $U$ test was used to compare differences $(\mathbf{c}, \mathbf{d}, \mathbf{e})$. Error bar represents the standard deviation. n.s. not statistically significant. * or ${ }^{\dagger}, p<0.05$

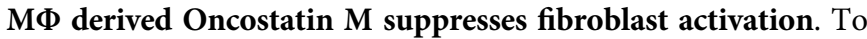
elucidate the molecular mechanisms by which hypoxic $M \Phi$ regulate fibroblast activation, we established an in vitro assay system using $\mathrm{C} 3 \mathrm{H} / 10 \mathrm{~T} 1 / 2$ cells, a mouse embryo fibroblast cell line, and thioglycollate-elicited peritoneal macrophages (TEPMs). TGF- $\beta 1$ induces $\alpha S M A$ mRNA expression in C3H/10T1/2 cells, which is known as an activation marker of fibroblasts. While the culture supernatant of TEPMs kept under normoxic conditions did not alter the expression of $\alpha S M A$ mRNA, the culture supernatant of TEPMs kept under hypoxic conditions significantly suppressed $\alpha S M A$ mRNA expression (Fig. 3a). Based on this finding, we hypothesized that hypoxia stimulates the secretion of some unknown factors in TEPMs, which suppress fibroblast activation.

To identify the hypoxia-stimulated secretion factors that have anti-fibrotic potential, we isolated primary TEPMs from hematopoietic/endothelial-specific HIF-1a knockout mice (HIF-1 $1 \alpha^{f l o x} / f l o x$; Tie2-cre ${ }^{+/-}$mice; HIF-1a KO) or cre negative littermate controls. Subsequently, we performed transcriptome analysis and searched for any secretory factors that are highly expressed in hypoxic conditions through a HIF-1 $\alpha$-dependent manner (Fig. 3b, left). This unbiased approach revealed 11 secretory factors, including Connective tissue growth factor (Ctgf), Chemokine (C-X-C motif) ligand 2 (Cxcl2), Cxcl3, Leukemia inhibitory factor (Lif) and Osm (Fig. 3b, right). To test their effects on fibroblast activation, we treated $\mathrm{C} 3 \mathrm{H} / 10 \mathrm{~T} 1 / 2$ cells with 10 of the identified secretory factors those we could obtain on a commercial basis, and tested their effects on fibroblast activation. Through this approach, we discovered that OSM, a member of the IL6-type family of cytokines, significantly suppressed $\alpha S M A$ mRNA expression in C3H/10T1/2 cells (Fig. 3c, Supplementary Fig. 13). OSM also suppressed the activation of isolated mouse primary cardiac fibroblasts (Fig. 3d). We further tested the expression of OSM in the heart and identified that OSM is highly expressed in murine cardiac MФ (Fig. 3e).

OSM is induced in hypoxia through a HIF-1a dependent manner. We cultured wild type TEPMs or bone marrow derived macrophages under $1 \%$ oxygen concentration and found that the Osm mRNA levels were significantly elevated under hypoxia (Fig. 4a, Supplementary Fig. 14). Hypoxia elicited elevation of Osm gene expression was significantly suppressed in HIF-1a KO TEPMs, indicating that the abundance of Osm mRNA is increased in a HIF-1 $\alpha$-dependent manner. To examine the molecular mechanisms by which HIF-1 $\alpha$ induces Osm gene expression, we next performed chromatin immunoprecipitation (ChIP) assay with anti-HIF-1a antibody. The results showed the direct binding of HIF- $1 a$ to the HRE sequence $4 \mathrm{~kb}$ upstream of the Osm transcription start site (Fig. 4b). To evaluate the roles of Osm HRE sequence in its transcriptional activation, we generated a reporter construct containing the HRE sequence of Osm. Co- 
a

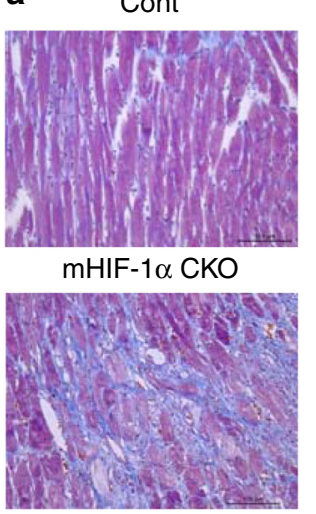

b

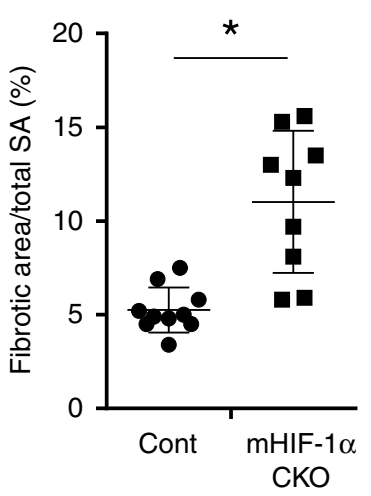

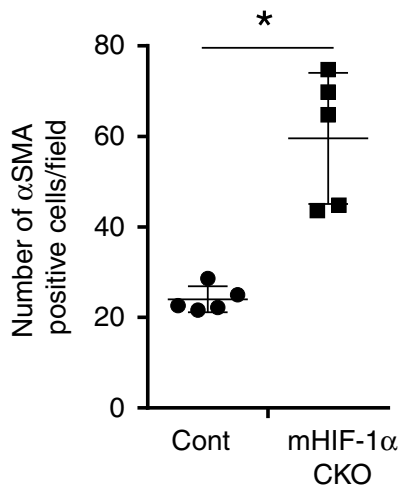

c

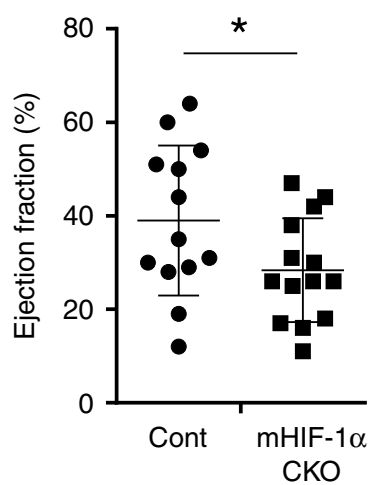

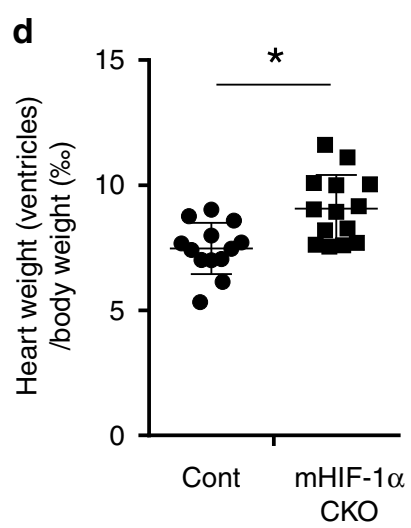

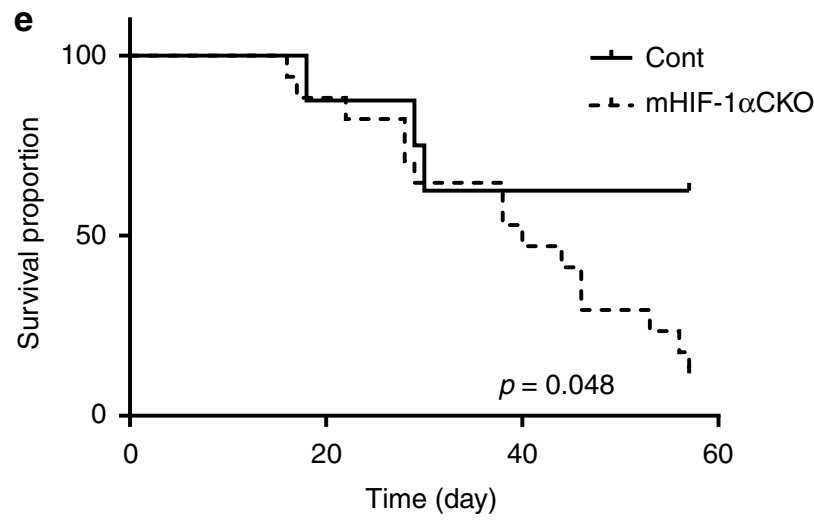

Fig. 2 Cardiac fibrosis develops in myeloid-specific HIF-1 $\alpha$ conditional knockout mice. a Masson's trichrome staining was performed using cardiac tissue of myeloid-specific HIF-1 $\alpha$ conditional knockout mice ( $m$ HIF- $1 \alpha$ CKO: HIF- $1 \alpha^{\text {flox/flox; }}$ LysM-cre ${ }^{+/}$) 14 days after TAC operation. Cre negative littermates were used as a control (cont). Fibrotic area was calculated compared to the total surface area (SA). Cont $(n=10)$ and $\mathrm{mHIF}-1 \alpha \mathrm{CKO}(n=9)$. Scale bar $=100$ $\mu \mathrm{m} . \mathbf{b}$ The average number of $\alpha$-smooth muscle actin ( $\alpha$ SMA) positive cells 14 days after TAC operation were counted within five fields. Cont $(n=5)$ and mHIF-1 $\alpha$ CKO $(n=5)$. c Data represent the ejection fraction 28 days after TAC operation in cont $(n=13)$ and mHIF-1 $\alpha$ CKO mice $(n=14)$. $\mathbf{d}$ Data represent the heart weight (ventricles) per body weight (\%o 28 days after TAC operation in cont $(n=13)$ and mHIF-1 $\alpha$ CKO mice $(n=14)$. The Mann-Whitney $\mathrm{U}$ test was used to compare differences between $\mathrm{mHIF}-\alpha \mathrm{CKO}$ and cont $(\mathbf{a}, \mathbf{b}, \mathbf{c}, \mathbf{d})$. e The Kaplan-Meier survival curves for control mice $(n$ $=8)$ and mHIF- $1 \alpha$ CKO mice $(n=17)$. The log rank test was used for the statistical analysis. Error bar represents the standard deviation. ${ }^{\star} p<0.05$

transfection of HIF-1 $\alpha$ and aryl hydrocarbon receptor nuclear translocator significantly enhanced the luciferase activity of the reporter construct containing Osm HRE sequences (Supplementary Fig. 15). Together, these results demonstrate that Osm expression is directly induced in hypoxia through a HIF-1adependent manner.

OSM inhibits SMAD signaling pathway. We further investigated the molecular processes by which OSM decreases $\alpha S M A$ mRNA expression level in $\mathrm{C} 3 \mathrm{H} / 10 \mathrm{~T} 1 / 2$ cells and primary cardiac fibroblasts. While OSM suppressed TGF- $\beta 1$ mediated augmentation of $\alpha S M A$ mRNA expression, IL6 did not affect its abundance (Fig. 5a, Supplementary Fig. 16), suggesting that the antifibrotic effect is specific to OSM and not a general feature of the IL6 cytokine family. TGF- $\beta 1$ signaling is mainly mediated through a canonical SMAD signaling pathway. It has been shown that extracellular signal-regulated kinase $1 / 2$ (ERK1/2) mediated phosphorylation of the SMAD linker region suppresses its nuclear translocation ${ }^{33-37}$. In this study, we found that OSM activates ERK signaling in $\mathrm{C} 3 \mathrm{H} / 10 \mathrm{~T} 1 / 2$ cells (Fig. 5b). Furthermore, OSM elicits the phosphorylation of SMAD2 linker region (Ser245/250/ 255), which was abolished by U0126, a selective MEK1/2 inhibitor. Notably, IL6 does not affect the phosphorylation of SMAD2 linker region. Next we extracted nuclear fractions and examined SMAD2 C-terminal (Ser465/467) phosphorylation, an activation marker of SMAD2 signaling. OSM significantly suppressed the accumulation of activated SMAD2 in the nucleus (Fig. 5c). Consistent with our hypothesis, U0126 attenuated the inhibitory effect of OSM on $\alpha S M A$ gene expression in $\mathrm{C} 3 \mathrm{H} / 10 \mathrm{~T} 1 / 2$ cells and primary cardiac fibroblasts (Fig. 5d, Supplementary Fig. 17). These results indicate that OSM exerts its anti-fibrotic effect partly through ERK1/2-mediated phosphorylation of the SMAD2 linker region.

OSM suppresses cardiac fibrosis in vivo. It should be noted that OSM suppressed fibroblast activation, whereas IL6 did not. While OSM and IL6 share gp130 as a co-receptor for the signaling, murine OSM receptor (OSMR) is specifically activated by OSM but not IL6. To test the effect of OSM on cardiac fibroblast in vivo, we generated fibroblast-specific Osmr knockout mice (OSMRflox/flox; Colla1-creERT, fOSMR CKO $)^{38}$. The extent of baseline cardiac fibrosis or heart weight remain unchanged in fOSMR CKO mice (Supplementary Fig. 18). We next performed murine TAC operation in fOSMR CKO mice and cre-negative littermates or tamoxifen untreated mice as controls. Cardiac fibrosis was more prominent in fOSMR CKO mice than controls (Fig. 6a). To further determine the roles of OSM in vivo, we injected OSM neutralizing antibody and measured the area of cardiac fibrosis in the murine TAC model. The results showed that inhibition of OSM significantly augments the development of 
a

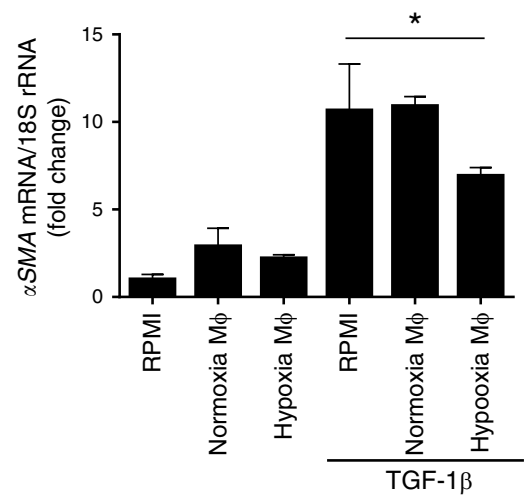

TGF- $\beta 1$ (2.5 ng per ml)

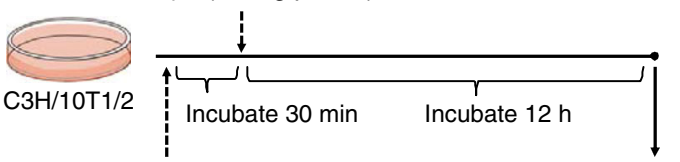

- RPMI

aSMA mRNA analysis

- Supernatant of TEPMs normoxia

- Supernatant of TEPMs hypoxia (1\%)

b

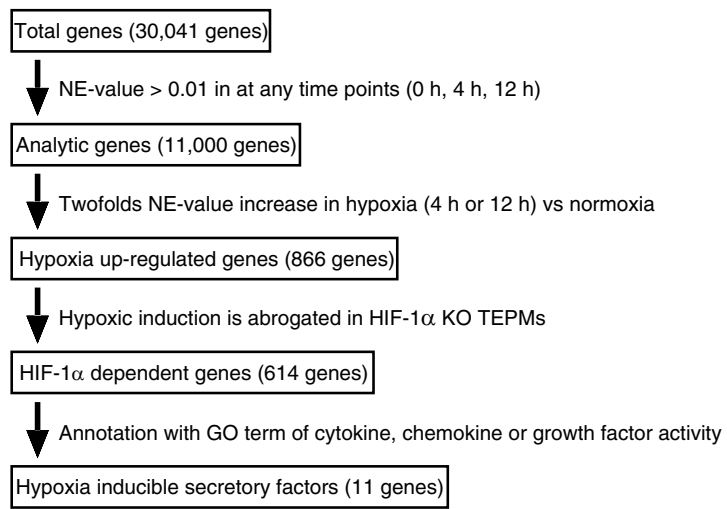

1. Connective tissue growth factor (Ctgf)
2. Chemokine (C-X-C motif) ligand $2(C x c / 2)$
3. Chemokine (C-X-C motif) ligand $3(C x c / 3)$
4. Leukemia inhibitory factor ( Lif)
5. Oncostatin M (Osm)
6. Heparin-binding EGF-like growth factor (Hbegf)
7. Tissue inhibitor of metalloproteinase 1 (Timp1)
8. CKLF-like MARVEL transmembrane domain containing 4
(Cmtm4)
9. Chemokine (C-X-C motif) ligand 1 (Cxc/1)
10. Mesencephalic astrocyte-derived neurotrophic factor (Manf)
11. Jagged 1 (Jag1)

C

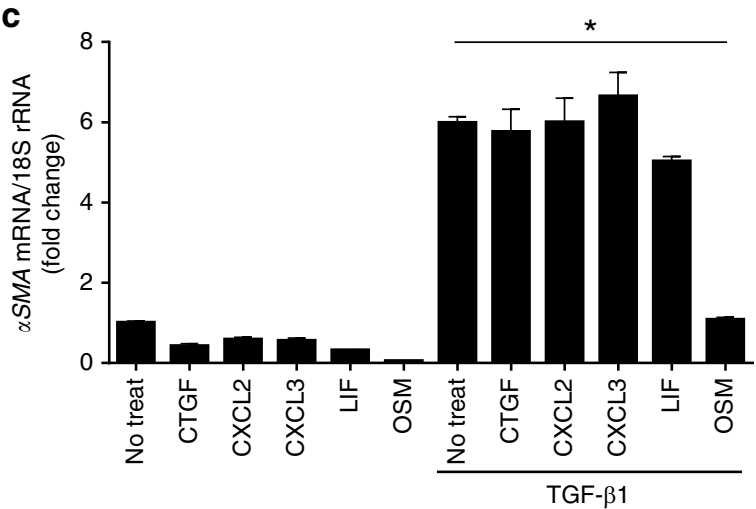

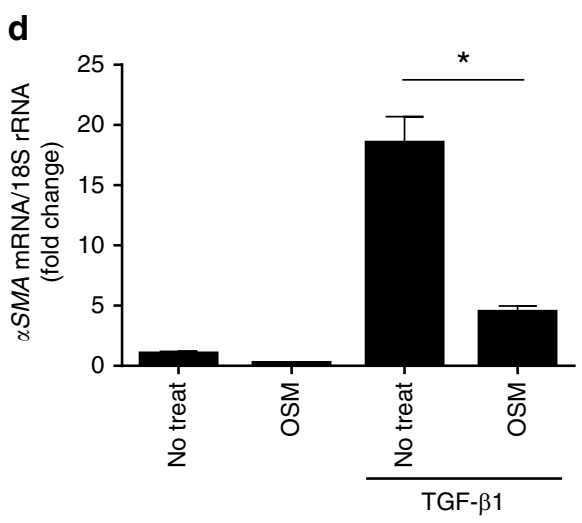

e

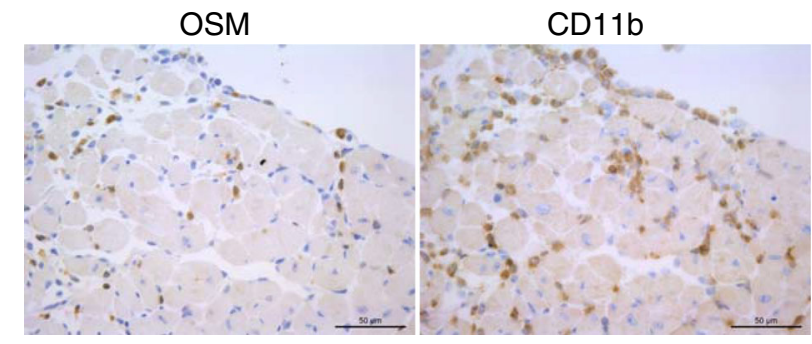

cardiac fibrosis (Fig. 6b). Collectively, these data suggest that OSM directly suppresses the activation of cardiac fibroblasts both in vitro and in vivo. Finally, we examined the expression of OSM in human heart specimens, which were obtained from heart failure patients. Notably, the number of OSM-positive cells in human heart specimen inversely correlated with the area of cardiac fibrosis (Fig. 6c, Supplementary Fig. 19), which is consistent with the anti-fibrotic function of OSM.

\section{Discussion}

Cardiac fibroblasts are activated not only in myocardial infarction, but also in pressure-overload-induced cardiac hypertrophy. Despite the fact that $M \Phi$ could suppress cardiac fibrosis 9 , its regulatory signals have not been fully elucidated. Here, we used a hypoxia probe in vivo, and demonstrated that tissue hypoxia develops in pressure-overload-induced cardiac remodeling. We further discovered that hypoxic $M \Phi$, but not normoxic $M \Phi$, 
Fig. 3 Oncostatin $M$ from hypoxic $M \Phi$ inhibits fibroblast activation. a Supernatants were collected from thioglycollate-elicited peritoneal macrophages (TEPMs) under normoxic or hypoxic condition. After pretreatment with the supernatants, $\mathrm{C} 3 \mathrm{H} / 10 \mathrm{~T} 1 / 2$ cells were stimulated with TGF- $\beta 1$ (2.5 ng per ml, $12 \mathrm{~h}$ ) and the relative expression of $\alpha$ SMA mRNA was calculated (right). The one-way ANOVA and Dunnett's multiple comparisons test were used for the statistical analysis $(F(2,6)=6.019)$. b We performed transcriptome analysis in isolated primary TEPMs from hematopoietic/endothelial-specific HIF-1 $\alpha$

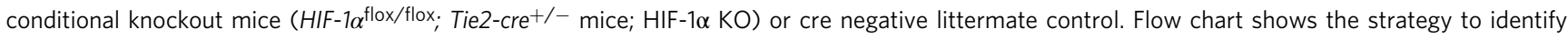
hypoxia-inducible secretory factors (left). Table illustrates the identified 11 hypoxia-inducible secretory factors (right). c The effect of each hypoxic inducible secretory factor in fibroblasts activation was examined ( $20 \mathrm{ng}$ per $\mathrm{ml}$ for CTGF, CXCL2, CXCL3, LIF and $10 \mathrm{ng}$ per ml for OSM). Thirty minutes after pretreatment with hypoxia-inducible secretory factors, C3H/10T1/2 cells were stimulated with TGF- $\beta 1(2.5 \mathrm{ng}$ per $\mathrm{ml}, 12 \mathrm{~h})$ and the relative expression of $\alpha$ SMA mRNA was calculated. The one-way ANOVA and Dunnett's multiple comparisons test were used for the statistical analysis ( $F(5,12)$ =68.07). $\mathbf{d}$ The effect of oncostatin M (OSM) (10 ng per $\mathrm{ml})$ in primary cardiac fibroblasts activation was examined. Thirty minutes after pretreatment with OSM (10 ng per $\mathrm{ml}, 12 \mathrm{~h}$ ), C3H/10T1/2 cells were stimulated with TGF- $\beta 1$ ( $2.5 \mathrm{ng}$ per $\mathrm{ml}, 12 \mathrm{~h}$ ) and the relative expression of $\alpha \mathrm{SMA} \mathrm{mRNA}$ was calculated. Two-tailed $t$-test with Welch's correction was used for the statistical analysis $(t=10.89, \mathrm{df}=2.208)$. Data show the mean and the standard deviation (error bar) of technical triplicates from a representative experiment. e Immunohistological staining were performed using cardiac tissues from TAC operated mice (day 3). OSM (left), CD11b (right). Scale bar $=50 \mu \mathrm{m}$. ${ }^{\star} p<0.05$
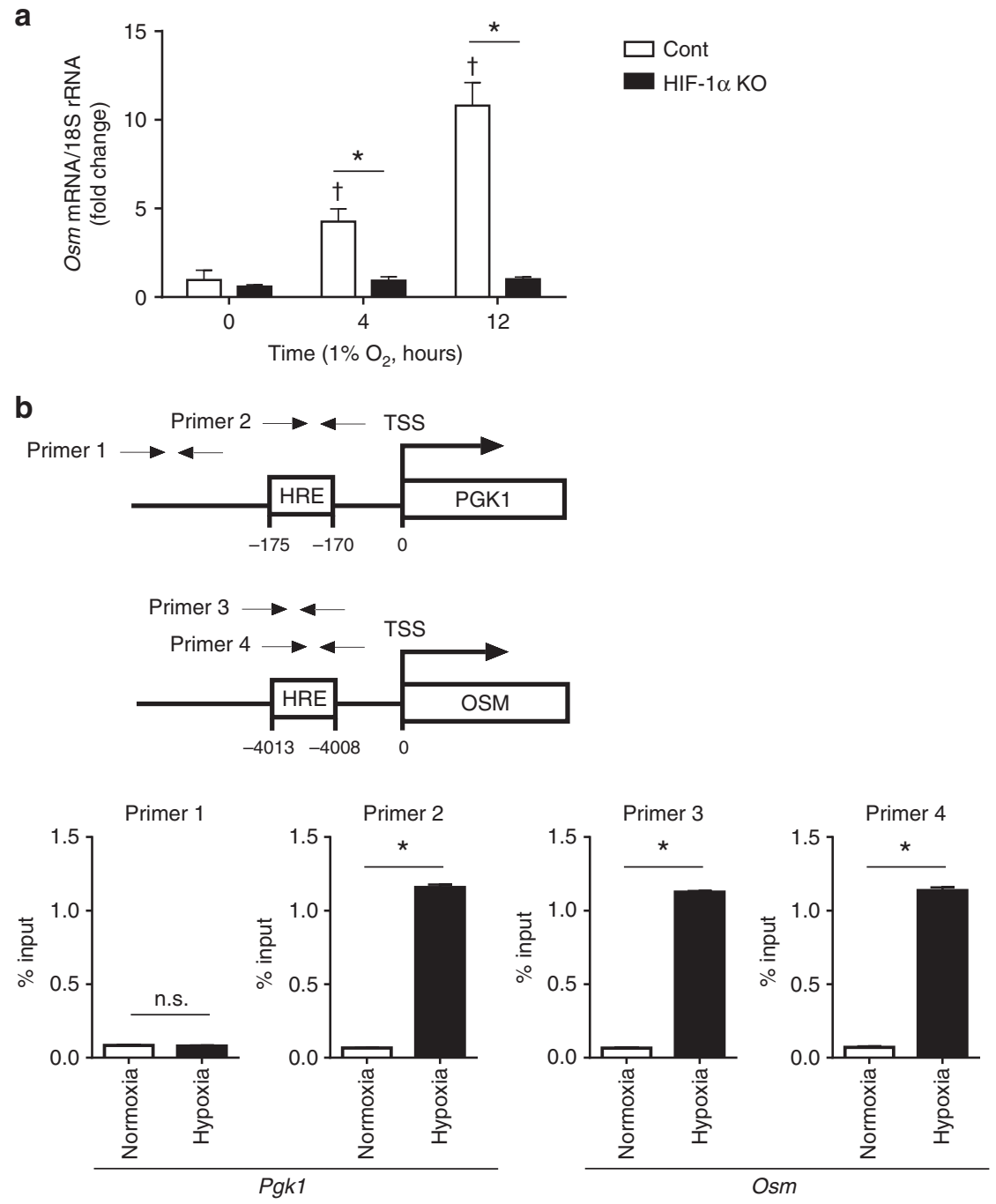

Fig. 4 OSM gene expression is induced in hypoxia through a HIF-1 $\alpha$ dependent manner. a Thioglycollate-elicited peritoneal macrophages (TEPMs) were isolated from hemtopoietic/endothelial-specific HIF-1 $\alpha$ knockout mice (HIF-1 $\alpha \mathrm{KO}$ ) or cre negative littermates as a control (cont). The TEPMs were exposed to hypoxia $\left(1 \% \mathrm{O}_{2}\right)$, and the relative expression level of Osm mRNA was analyzed. The one-way ANOVA and Dunnett's multiple comparisons test were used for the statistical analysis $(F(2,6)=90.20) .{ }^{\dagger} p<0.05$ vs $0 \mathrm{~h}$. The two-way ANOVA and Sidak's multiple comparison test was performed for the statistical analysis $(F(2,12)=94.66) .{ }^{*} p<0.05$ vs cont. $\mathbf{b} \mathrm{HIF}-1 \alpha$ binding to the promoter of Osm gene was studied by chromatin immunoprecipitation coupled to detection by quantitative PCR. Primer set 1 and 2 were designed to detect the promoter legion ( -500 and -170 bp) of Pgk1 gene. Primer set 3 and 4 were designed to detect the hypoxia response element (HRE, $-4 \mathrm{~kb}$ ) of Osm gene. Data show the mean and the standard deviation (effort bar) of technical triplicates from a representative experiment. Quantitative PCR analysis were repeated at least three independent experiments. Two-tailed $t$-test with Welch's correction was used for the statistical analysis (Primer $1(t=1.527, d f=3.863)$, Primer $2(t=93.69, d f=2.011)$, Primer $3(t=197.7, d f=$ 3.202), Primer $4(t=75.41, d f=2.505))$. n.s. not statistically significant. ${ }^{*} p<0.05$ 
a

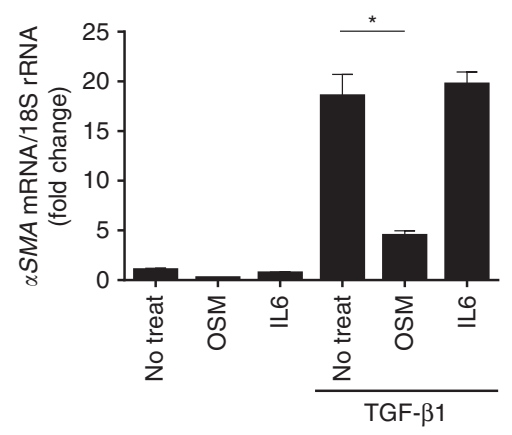

C

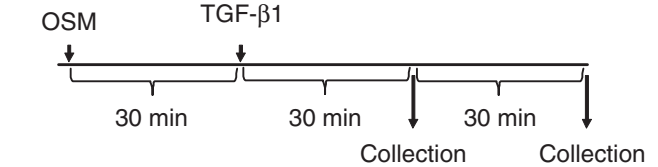

b

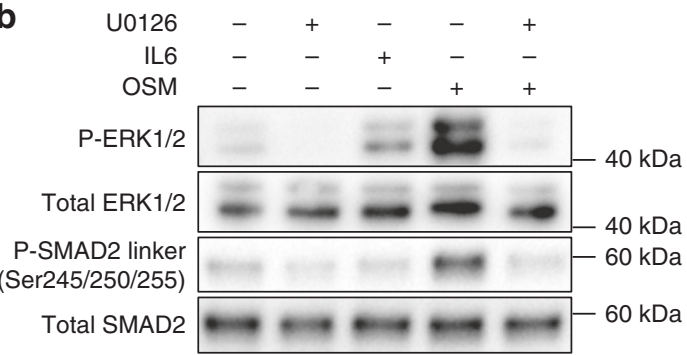

d

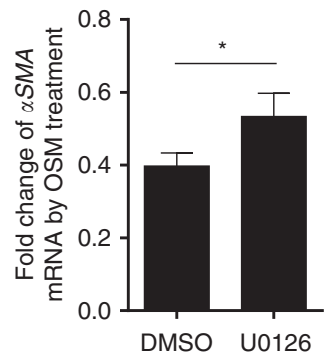

Fig. 5 OSM suppresses SMAD signaling. a After pretreatment with the OSM (10 ng per ml) or IL6 (20 ng per ml), C3H/10T1/2 cells were stimulated with TGF- $\beta 1$ ( $2.5 \mathrm{ng}$ per $\mathrm{ml}, 12 \mathrm{~h}$ ) and the relative expression level of $\alpha$ SMA mRNA was calculated. The one-way ANOVA and Dunnett's multiple comparisons test were used for the statistical analysis $(F(2,6)=97.35)$. b Immunoblot analysis of phospho-ERK1/2, total ERK1/2, phospho-SMAD2 linker (Ser245/ 250/255) and total SMAD2 protein. Thirty minutes after U0126 (20 nM) pretreatment, C3H/10T1/2 cells were stimulated with OSM (10 ng per ml) or IL6 (20 $\mathrm{ng}$ per $\mathrm{ml}$ ) for $30 \mathrm{~min}$. and collected for the analysis. U0126: a selective MEK1/2 inhibitor. c Immunoblot analysis of phospho-SMAD2 C-terminal (Ser465/467) and Lamin A/C. Thirty minutes after OSM (10 ng per ml) pretreatment, C3H/10T1/2 cells were stimulated with TGF- $\beta 1$ ( $2.5 \mathrm{ng}$ per ml, $12 \mathrm{~h}$ ) and collected at indicated time points. d After pretreatment with $\mathrm{U} 0126(20 \mu \mathrm{M}, 60 \mathrm{~min})$ and OSM (10 $\mathrm{ng}$ per $\mathrm{ml}, 30 \mathrm{~min}), \mathrm{C} 3 \mathrm{H} / 10 \mathrm{~T} 1 / 2 \mathrm{cells}$ were stimulated with TGF- $\beta 1$ ( $2.5 \mathrm{ng}$ per $\mathrm{ml}, 12 \mathrm{~h}$ ) and the relative expression level of $\alpha$ SMA mRNA was calculated. Two-tailed $t$-test with Welch's correction was used for the statistical analysis $(t=3.212, d f=3.172)$. Data show the mean and the standard deviation (error bar) of technical triplicates from a representative experiment $(\mathbf{a}, \mathbf{d}) .{ }^{*} p<0.05$

suppresses fibroblast activation through the secretion of OSM. While both OSM and IL6 transduce their signals through gp130, OSMR specifically interact with src homology 2 domaincontaining transforming protein $\mathrm{C} 1(\mathrm{SHC} 1)^{39}$. This seems to account for the OSM mediated activation of ERK signaling, contributing to its anti-fibrotic properties. OSM was also shown to have a protective effect in ischemic myocardium ${ }^{40,41}$, thus Ly6C ${ }^{\text {hi }} M \Phi$-derived OSM exerts a cardio-protective effect on both cardiomyocytes and cardiac fibroblasts. Our results demonstrate a functional link between sterile inflammation and cardiac fibrosis.

In myocardial infarction model, Ly6Chi and $\mathrm{Ly}^{\text {hi }} \mathrm{C}^{\text {lo }} \mathrm{M} \Phi$ sequentially accumulate in the heart ${ }^{9}$. It has also been shown that CCL2 or Regenerating islet-derived $3 \beta(\operatorname{Reg} 3 \beta)$ has a strong potential to recruit $\mathrm{Ly} 6 \mathrm{C}^{\text {hi }} \mathrm{M} \Phi$ to the damaged heart ${ }^{42}$. In our model, the expression of $C c l 2$, but not Reg3 $\beta$ is highly induced at day 3 after TAC (Supplementary Fig. 20). While it is uncertain whether Ly6Chi and Ly $6 C^{\text {lo }} \mathrm{M} \Phi$ give rise to distinct macrophage subsets in the heart, the results of our study are consistent with the finding that $\mathrm{Ly} 6 \mathrm{C}^{\mathrm{hi}} \mathrm{M} \Phi$ are recruited to the damaged heart through the CCL2-CCR2 axis 9 .

The results of this study have revealed an anti-fibrotic effect of OSM (Supplementary Fig. 21). We still need to be careful in using OSM or an OSMR agonist for the management of cardiac fibrosis, since it may have a potential to drive intestinal inflammation ${ }^{43}$. Therefore, selective delivery of OSM to the heart such as coronary artery injection seems to be necessary to manage cardiac fibrosis effectively. It should also be noted that the fibroblast activation processes in TAC model may be quite different from that in myocardial infarction. Further investigation, therefore, will help to understand the context dependent activation processes of cardiac fibrosis in more detail.

\section{Methods}

Mice and surgical procedures. All animal experiments were approved by the University of Tokyo Ethics Committee for Animal Experiments or King's College London Ethical Review Process Committee and UK Home Office (Project License No. PPL70/7260). The procedures are strictly adhered to the guidelines for animal experiments of the University of Tokyo or the Guidance on the Operation of the Animals (Scientific Procedures) Act, 1986 (UK Home Office). C57BL/6 mice were purchased from Crea Japan (Tokyo, Japan) or Envigo (Blackthorn, UK). B6.129Hifla ${ }^{\text {tm } 3 \text { Rsjo/J }}$ (HIF-1 $\left.\alpha^{\text {flox/fox }}\right)$ mice, B6;129-Osmr ${ }^{\text {tml.1Nat/J }}\left(\right.$ OSMR $\left.^{\text {flox/flox }}\right)$ mice,

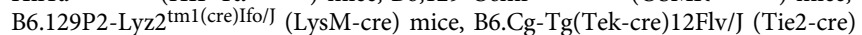
mice and B6.Cg-Tg(Collal-cre/ERT2)1Crm/J (Colla1-creERT) mice were purchased from The Jackson Laboratory (Bar Harbor, Maine, USA). HIF-1 $a^{\text {flox/fox }}$, LysM-Cre and Tie2-cre mice were backcrossed to C57BL/6 background. Mice were housed in a specific pathogen-free facility with a 12 -h light/12-h dark cycle. Tamoxifen (1 mg) (Sigma-Aldrich, St-Louis, USA) was injected intraperitoneally for 7 days to induce cre-mediated recombination in Colla1-creERT mice. Corn oil was administered in control groups. Seven days following cessation of tamoxifen animals were subjected to TAC procedure. Male mice 7-12 weeks of age were used for TAC operation. TAC operation was performed as follows, a 27-gauge needle was placed next to the aortic arch, the suture was tied firmly two times around the 27 -gauge needle and the aorta, and the needle was removed ${ }^{34}$. Sham operated mice underwent the same procedure but without constriction. Mice were closely observed and euthanized quickly, at a humane end point (no locomotion or body weight loss exceeding $20 \%$ of the initial body weight). 
a
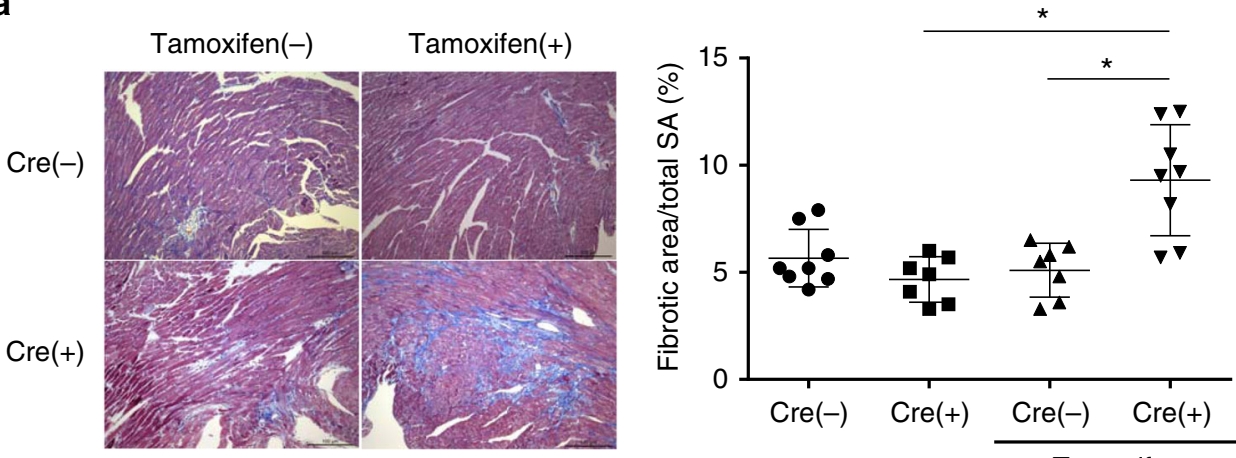

Tamoxifen

b

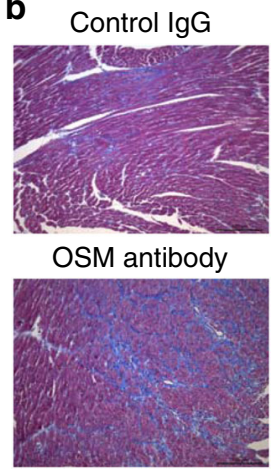

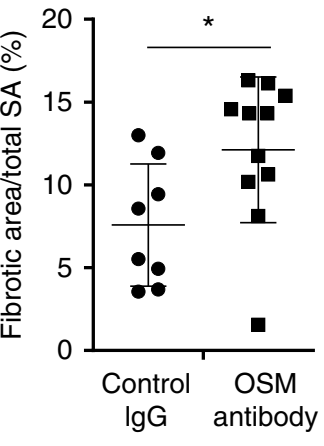

C

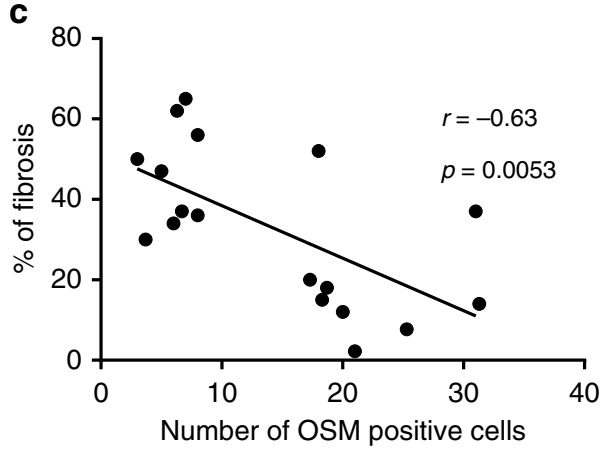

Fig. 6 OSM suppresses cardiac fibrosis in vivo. Masson's trichrome staining was performed using cardiac tissue of fibroblast-specific Osmr knockout mice (OSMRflox/flox; Colla1-creERT, Cre(+)) 14 days after TAC operation. Cre negative littermates $(C r e(-))$ or tamoxifen untreated littermates were used as controls. Fibrotic area was calculated compared to the total surface area (SA) (right). The Kruskal-Wallis test was used for the statistical analysis. (Cre( - ), tamoxifen(-): $n=8, \operatorname{Cre}(+)$, tamoxifen(-): $n=7$, Cre(-), tamoxifen(+): $n=7, \operatorname{Cre}(+)$, tamoxifen( +$): n=8)$. Scale bar $=100 \mu$ m. b The effect of neutralizing antibody to OSM on cardiac fibrosis was examined. Neutralizing antibody to OSM (100 $\mu \mathrm{g})$ or control lgG (100 $\mu \mathrm{g})$ was injected intravenously 3 days after TAC operation. Masson's trichrome staining was performed using cardiac tissue 14 days after TAC operation. Fibrotic area was calculated compared to the total surface area (SA). The Mann-Whitney $U$ test was used to compare differences between the control IgG $(n=8)$ group and OSM antibody group $(n=11)$. Data show the mean and the standard deviation (error bar). Scale bar $=100 \mu \mathrm{m}$. $\mathbf{c}$ The correlation between the area of cardiac fibrosis and the number of OSM-positive cells was examined. Linear regression analysis was used for the correlation between percent of fibrotic area and the number of OSM-positive cells in the heart specimens from human heart failure patients $(n=18) .{ }^{\star} p<0.05$

Cells, reagents, recombinant proteins, and antibodies. Mesenchymal stem cell line C3H10T1/2 cells were purchased from American Type Culture Collection. C3H10T1/2 cells were grown in DMEM (Dulbecco's Modified Eagle Medium) medium containing $10 \%$ fetal bovine serum (HyClone, GE Healthcare Japan, Tokyo, Japan) in a $37^{\circ} \mathrm{C}$ incubator $\left(5 \% \mathrm{CO}_{2}\right)$. TEPMs were collected from the peritoneal cavity 4 days after the intraperitoneal injection of $3 \%$ thioglycollate solution (Fluka, Sigma-Aldrich, St Louis, MO, USA) using female mice. TEPMs were grown in RPMI1640 (GIBCO, Life technologies, Carlsbad, CA, USA) medium containing $10 \%$ fetal bovine serum (HyClone) in a $37^{\circ} \mathrm{C}$ incubator $\left(5 \% \mathrm{CO}_{2}\right)$. Adult male mice at the age of 6-8 weeks were used for the isolation and cultivation of cardiac fibroblasts ${ }^{44}$. Two or three hearts of ventricles were isolated and quickly minced into small pieces. After incubation at $37^{\circ} \mathrm{C}$ for 30 min with DMEM containing Liberase TH ( $25 \mu \mathrm{g}$ per ml, Sigma-Aldrich) and elastase (1.2 U per ml, Worthington Biochemical Corporation, Freehold, NJ, USA), the cell suspensions were pelleted and washed with PBS(-). All cell suspensions were plated on a collagen-coated dish (Cosmo Bio, Tokyo, Japan) in DMEM supplemented with $10 \%$ of fetal bovine serum. After overnight incubation, nonadherent cells were removed, and adherent cells were cultivated. Primary fibroblasts at passage 1 or 2 were used for experiments.

Recombinant mouse OSM (catalog no. 495-MO-025), mouse IL6 (catalog no. 406-ML-005), and human TGF- $\beta 1$ (catalog no. 240-B-010) were all purchased from R\&D Systems (Minneapolis, MN, USA). Recombinant mouse CTGF (catalog no. RPA010MU01) was purchased from Cloud-Clone Corp. (Katy, TX, USA). Recombinant mouse CXCL2 (catalog no. 582502), mouse LIF (catalog no 554002) and mouse CXCL3 (catalog no. 590802) were purchased from Bio Legend (San Diego, CA, USA). All recombinant proteins used in cell culture were diluted in $0.5 \%$ BSA solution (Sigma, catalog no. A8806-1G).

APC-CD11b (M1/70) (diluted 1:1000, 17-0112-82; eBioscience, PE-F4/80 (BM8) (diluted 1:1000, 123110; BioLegend, FITC-Ly6C (HK1.4) (diluted 1:1000, 128006; BioLegend, Pacific Blue-Ly6G (1A8) (diluted 1:1000, 127612; BioLegend) were used for the FACS analysis. Mouse OSM antibody (AF-495-NA; R\&D) or normal Goat IgG control (AB-108-C; R\&D) was used as a neutralizing antibody or control IgG in vivo. Human OSM antibody (diluted 1:500, NBP1-87768; R\&D) and mouse OSM antibody (diluted 1:500, bs-5095R; BIS) were used for the OSM staining in human and mouse heart tissues respectively. Mouse CD11b antibody (diluted 1:2000, ab133357; Abcam, Cambridge, UK) was used for the CD11b staining in mouse heart tissues.

Exposure of cell cultures to hypoxia $\left(1 \% \mathrm{O}_{2}\right)$ was performed in the hypoxic workstation In $\mathrm{Vivo}_{2} 500$ (Ruskinn Technology, Bridgend, UK). The serum free culture medium of TEPMs was changed $12 \mathrm{~h}$ after hypoxic exposure. Follow which, TEPMs were exposed to hypoxia for $12 \mathrm{~h}$, and their supernatant was collected and used in the analysis of fibroblast activation.

Preparation of clodronate liposomes. Clodronate liposomes were generated as follows ${ }^{45}$. After $8 \mathrm{mg}$ cholesterol was dissolved in $10 \mathrm{ml}$ chloroform, $0.86 \mathrm{ml}$ of phosphatidylcholine solution (containing $86 \mathrm{mg}$ phosphatidylcholine) was added. The chloroform phase was removed by low vacuum rotation $(1.257 \times g)$ evaporation. The phospholipid film was dispersed in $10 \mathrm{ml}$ of aqueous solution containing $0.6 \mathrm{M}$ clodronate by gentle rotation at room temperature. The milky white suspension was kept at room temperature for $2 \mathrm{~h}$ under nitrogen gas. The suspension was sonicated in a waterbath sonicator for $3 \mathrm{~min}$ and kept overnight at $4{ }^{\circ} \mathrm{C}$. After centrifugation $(10,000 \times g$ for $15 \mathrm{~min})$, the solution under the white band of liposomes was removed using a Pasteur pipette. The clodronate liposomes are washed three times using sterilized PBS (Centrifugation at $25,000 \times g$ for $30 \mathrm{~min}$ ). Finally, the pellet was resuspended in $4 \mathrm{ml}$ sterilized PBS.

Flow cytometry. All flow cytometric analyses and sorting were performed using FACS aria II (BD) and FlowJo software (Tree Star). To isolate cells from hearts, whole heart was cut into small pieces in DMEM (containing 10\% fetal bovine serum). The collected tissue was incubated for $2 \mathrm{~h}$ with collagenase type I (1 mg per $\mathrm{ml}$, Worthington Biochemical Corporation) and elastase $(0.74 \mathrm{U}$ per $\mathrm{ml}$, Worthington Biochemical Corporation) in Hanks' buffered saline solution (HBSS). The suspension was passed through a 22-gauge needle and washed in ice-cold PBS(-) 
containing 5\% fetal bovine serum. After removing any erythrocytes using BD PharmLyse (BD), the samples were subjected to flow cytometric analysis.

The heart samples were obtained $45 \mathrm{~min}$. after intraperitoneal injection of pimonidazole (Pimo, $60 \mathrm{mg}$ per $\mathrm{kg}$ ) (hpi, Burlington, MA, USA). Intracellular incorporation of Pimo was analyzed by multicolor FACS with a fluorescein (FITC)conjugated anti-Pimo antibody (diluted 1:1000, hpi)

Intravital visualization of hypoxic signals in heart. Hypoxic state is visualized using two photon microscope and phosphorescence probe, LOX-1 (Scivax Life Sciences, Tokyo, Japan $)^{46}$. Anesthesized and mechanically-ventilated mice were administrated with Hoechst 33342 (10 mg per kg, Invitrogen (Thermofisher), blue), fluorescein labeled Griffonia Simplicifolia Lectin I (GSL I) isolectin B4 (green, Vector laboratories, FL-1201, $50 \mu \mathrm{g}$ for each mouse), and LOX-1 hypoxic probes (100 $\mu \mathrm{g}$ for each mouse, red). Imaging of living heart was performed by two photon microscope (Nikon-A1RMP, Nikon), femtosecond infrared lasers (Chameleon Vision-II, Coherent), and $\times 40$ water-immersion objective lens (NA1.15, Nikon). Signal intensity for LOX-1 was automatically quantified and statistically analyzed by NIS-elements software (Nikon).

RNA isolation and quantitative RT-PCR. For gene expression analysis, total RNA was purified from cultured cells using RNeasy kits (Qiagen, Tokyo, Japan) according to the manufacturer's instructions. Complementary DNA was synthesized using the SuperScript III First-Strand Synthesis System (Life Technologies). Quantitative real-time PCR (RT-PCR) analyses were conducted using the LightCycler system (Roche Diagnostic, Tokyo, Japan), with 18S rRNA serving as the internal control. Primer sequences of the analyzed mouse genes are shown in Supplementary Table 1.

Western blotting. Cell extracts were separated on TGX FastCast acrylamide Kit, 7.5\% (BIO RAD Laboratories Inc.), and then transferred to PVDF membrane (Millipore, Bedford, MA, USA). After blocking in 5\% dry milk in Tris-buffered saline with Tween $20(0.1 \%)$ (TBS-T), the membrane was incubated with primary antibodies (1:1000) followed by incubation with horseradish peroxidase-conjugated secondary antibodies (1:2000). Detection was performed using ECL Prime (GE Healthcare, Logan, UT, USA) according to the manufacturer's instructions. The following antibodies were used: SMAD2 (\#5339, Cell Signaling Technology, Danvers, Ma, USA), Phospho-SMAD2 (Ser245/250/255) (\#3104, Cell Signaling Technology, Danvers, MA, USA), Phospho-SMAD2 (Ser465/467) (\#3108, Cell Signaling Technology, Danvers, MA, USA), Lamin A/C (\#2032, Cell Signaling Technology, Danvers, MA, USA), ERK (\#4695, Cell Signaling Technology, Danvers, MA, USA), Phospho-ERK (\#4370, Cell Signaling Technology, Danvers, MA, USA), goat anti-rabbit IgG ${ }^{-} \mathrm{HRP}$ (\#7074, Cell Signaling Technology, Danvers, MA, USA). After the exposure to phospho ERK1/2 or phosphor SMAD2 C-terminal antibody, the same membrane was exposed with anti-ERK or Lamin A/C antibody. Whole images of western blot images are shown in Supplementary Figs. 22-24.

ChIP assay. TEPMs were cross-linked for $10 \mathrm{~min}$ by $1 \%$ paraformaldehyde. After neutralization using $0.2 \mathrm{M}$ glycine solution, TEPMs were collected and suspended in SDS lysis buffer with cOmplete protease inhibitor cocktail (1873580, Roche). Samples were subjected to fragmentation using Sonifier (Branson, Dansbury, USA). Sonicated samples were immunoprecipitated with the HIF-1 $\alpha$ antibody (NB100134, Novus Biologicals). ChIP samples were quantified with RT-PCR using specific primer pairs (Supplementary Table 1).

RNA sequencing (RNA-seq) analysis. TEPMs were isolated from HIF- $1 \alpha \mathrm{KO}$ or cre negative littermate controls. We exposed TEPMs to hypoxia $\left(1 \% \mathrm{O}_{2}\right)$ or normoxia and collected total RNA at $4 \mathrm{~h}$ and $12 \mathrm{~h}$ time points, then performed transcriptome analysis (RNA-seq.). Single-end RNA-seq libraries were prepared using a TruSeq RNA Sample Prep Kit (Illumina, San Diego, CA). Sequencing runs were performed on an Illumina Genome Analyzer IIx (Illumina, San Diego, CA, USA). The reads were aligned to the $\mathrm{mm} 9$ mouse genome using TopHat (ver. 2.0.0). Generation of gene expression data, normalization and gene annotation processes were performed using Genomatix Genome Analyzer (Genomatix, Munich, Germany). The normalized expression value (NE-value) was calculated as follow: the number of reads per a gene $\times 10^{7}$ per the number of mapped reads in the genome $\times$ gene length.

Identification of hypoxia-inducible secretory factors. To identify hypoxiainducible secretory factors, we performed procedures as follows. Initially, we selected the genes with NE-value more than 0.01 in at any time points (Analytic genes: 11,000 genes). Second, we picked up the genes those maximum NE-vale were upregulated more than 2-folds in hypoxia (Hypoxia upregulated genes: 866 genes). Third, we selected the genes in which hypoxic induction of their expression is abrogated in HIF-1a KO TEPMs (HIF-1 $\alpha$ dependent genes: 614 genes)). Finally, genes that have gene ontology (GO) terms of 'cytokine activity', 'chemokine activity' or 'growth factor activity' were extracted (http://www.geneontology.org/) (Hypoxia-inducible secretory factors: 11 genes).
Histological studies of mouse and human heart tissues. Written informed consent was obtained from all participants. The study protocol was approved by the Ethics Committee of The Cardiovascular Institute (Tokyo, Japan), and strictly adhered to the ethical regulations. Heart tissues were obtained from heart failure patients, when they underwent heart surgery or endomyocardial biopsy. The patients with prior medical history of myocardial infarction were excluded. Immunohistochemistry were performed in each specimen. Masson trichrome staining and Sirius Red/ Fast Green staining were performed to calculate perivascular and interstitial fibrotic area.

Statistical analysis. All data are shown as average with SD. Comparison between two groups was analyzed using unpaired two-tailed $t$-test with Welch's correction. Differences among more than two groups were analyzed using one-way or two-way ANOVA followed by Dunnett's multiple comparisons test or Sidak's multiple comparison test or Kruskal-Wallis test. $P$-values of less than 0.05 were considered to be statistically significant. Kaplan-Meyer analysis, followed by a long-rank test, was used to analyze the survival of mice. Linear regression analysis was used for the correlation between percent of fibrotic area and the number of OSM-positive cells in the heart tissues. All statistical analyses were performed using Prism 6 for Windows (version 6.07, GraphPad, San Diego, CA, USA).

\section{Data availability}

All data contained in the manuscript are available from the corresponding author upon reasonable request. The RNA-seq data of peritoneal macrophages have been deposited in DDBJ BioProject under accession number DRA008230.

Received: 22 September 2017 Accepted: 5 June 2019 Published online: 27 June 2019

\section{References}

1. Khan, R. \& Sheppard, R. Fibrosis in heart disease: understanding the role of transforming growth factor-beta in cardiomyopathy, valvular disease and arrhythmia. Immunology 118, 10-24 (2006).

2. Sabbah, H. N., Sharov, V. G., Lesch, M. \& Goldstein, S. Progression of heart failure: a role for interstitial fibrosis. Mol. Cell Biochem 147, 29-34 (1995)

3. Fan, D., Takawale, A., Lee, J. \& Kassiri, Z. Cardiac fibroblasts, fibrosis and extracellular matrix remodeling in heart disease. Fibrogenes. Tissue Repair 5 15 (2012).

4. Lewis, G. A. et al. Biological phenotypes of heart failure with preserved ejection fraction. J. Am. Coll. Cardiol. 70, 2186-2200 (2017).

5. Assomull, R. G. et al. Cardiovascular magnetic resonance, fibrosis, and prognosis in dilated cardiomyopathy. J. Am. Coll. Cardiol. 48, 1977-1985 (2006).

6. Aoki, T. et al. Prognostic impact of myocardial interstitial fibrosis in nonischemic heart failure. -Comparison between preserved and reduced ejection fraction heart failure. Circ. J. 75, 2605-2613 (2011).

7. Gulati, A. et al. Association of fibrosis with mortality and sudden cardiac death in patients with nonischemic dilated cardiomyopathy. JAMA 309, 896-908 (2013).

8. Kuruvilla, S., Adenaw, N., Katwal, A. B., Lipinski, M. J., Kramer, C. M. \& Salerno, M. Late gadolinium enhancement on cardiac magnetic resonance predicts adverse cardiovascular outcomes in nonischemic cardiomyopathy: a systematic review and meta-analysis. Circ. Cardiovasc Imaging 7, 250-258 (2014)

9. Nahrendorf, M. et al. The healing myocardium sequentially mobilizes two monocyte subsets with divergent and complementary functions. J. Exp. Med. 204, 3037-3047 (2007).

10. Levine, B., Kalman, J., Mayer, L., Fillit, H. M. \& Packer, M. Elevated circulating levels of tumor necrosis factor in severe chronic heart failure. $N$. Engl. J. Med. 323, 236-241 (1990).

11. Mann, D. L. Inflammatory mediators and the failing heart: past, present, and the foreseeable future. Circ. Res. 91, 988-998 (2002).

12. Finkel, M. S., Oddis, C. V., Jacob, T. D., Watkins, S. C., Hattler, B. G. \& Simmons, R. L. Negative inotropic effects of cytokines on the heart mediated by nitric oxide. Science 257, 387-389 (1992)

13. Eltzschig, H. K. \& Carmeliet, P. Hypoxia and inflammation. N. Engl. J. Med. 364, 656-665 (2011).

14. Murdoch, C., Giannoudis, A. \& Lewis, C. E. Mechanisms regulating the recruitment of macrophages into hypoxic areas of tumors and other ischemic tissues. Blood 104, 2224-2234 (2004).

15. Wang, G. L. \& Semenza, G. L. General involvement of hypoxia-inducible factor 1 in transcriptional response to hypoxia. Proc. Natl Acad. Sci. USA 90, 4304-4308 (1993). 
16. Weidemann, A. \& Johnson, R. S. Biology of HIF-1alpha. Cell Death Differ. 15, 621-627 (2008).

17. Wang, G. L., Jiang, B. H., Rue, E. A. \& Semenza, G. L. Hypoxia-inducible factor 1 is a basic-helix-loop-helix-PAS heterodimer regulated by cellular $\mathrm{O} 2$ tension. Proc. Natl Acad. Sci. USA 92, 5510-5514 (1995).

18. Jiang, B. H., Semenza, G. L., Bauer, C. \& Marti, H. H. Hypoxia-inducible factor 1 levels vary exponentially over a physiologically relevant range of $\mathrm{O} 2$ tension. Am. J. Physiol. 271, C1172-C1180 (1996).

19. Kaelin, W. G. \& Ratcliffe, P. J. Oxygen sensing by metazoans: the central role of the HIF hydroxylase pathway. Mol. Cell 30, 393-402 (2008).

20. Semenza, G. L. Hypoxia-inducible factors in physiology and medicine. Cell 148, 399-408 (2012).

21. Bruick, R. K. \& McKnight, S. L. A conserved family of prolyl-4-hydroxylases that modify HIF. Science 294, 1337-1340 (2001).

22. Epstein, A. C. et al. C. elegans EGL-9 and mammalian homologs define a family of dioxygenases that regulate HIF by prolyl hydroxylation. Cell 107, 43-54 (2001).

23. Maemura, K. et al. Generation of a dominant-negative mutant of endothelial PAS domain protein 1 by deletion of a potent C-terminal transactivation domain. J. Biol. Chem. 274, 31565-31570 (1999).

24. Takeda, N. et al. Differential activation and antagonistic function of HIF\{alpha\} isoforms in macrophages are essential for NO homeostasis. Genes Dev. 24, 491-501 (2010).

25. Semba, H. et al. HIF-1alpha-PDK1 axis-induced active glycolysis plays an essential role in macrophage migratory capacity. Nat. Commun. 7, 11635 (2016).

26. Seki, E. et al. TLR4 enhances TGF-beta signaling and hepatic fibrosis. Nat. Med. 13, 1324-1332 (2007).

27. Swirski, F. K. et al. Ly-6Chi monocytes dominate hypercholesterolemiaassociated monocytosis and give rise to macrophages in atheromata. J. Clin. Invest 117, 195-205 (2007)

28. Nahrendorf, M. \& Swirski, F. K. Monocyte and macrophage heterogeneity in the heart. Circ. Res. 112, 1624-1633 (2013).

29. Hilgendorf, I. et al. Ly-6Chigh monocytes depend on Nr4a1 to balance both inflammatory and reparative phases in the infarcted myocardium. Circ. Res. 114, 1611-1622 (2014).

30. Takubo, K. et al. Regulation of the HIF-1alpha level is essential for hematopoietic stem cells. Cell Stem Cell 7, 391-402 (2010).

31. Cramer, T. et al. HIF-1alpha is essential for myeloid cell-mediated inflammation. Cell 112, 645-657 (2003).

32. Clausen, B. E., Burkhardt, C., Reith, W., Renkawitz, R. \& Förster, I Conditional gene targeting in macrophages and granulocytes using LysMcre mice. Transgenic Res. 8, 265-277 (1999).

33. Kretzschmar, M., Liu, F., Hata, A., Doody, J. \& Massagué, J. The TGF-beta family mediator Smad 1 is phosphorylated directly and activated functionally by the BMP receptor kinase. Genes Dev. 11, 984-995 (1997).

34. Kretzschmar, M., Doody, J., Timokhina, I. \& Massagué, J. A mechanism of repression of TGFbeta/ Smad signaling by oncogenic Ras. Genes Dev. 13, 804-816 (1999).

35. Yue, J., Frey, R. S. \& Mulder, K. M. Cross-talk between the Smad1 and Ras/MEK signaling pathways for TGFbeta. Oncogene 18, 2033-2037 (1999).

36. Gao, S. et al. Ubiquitin ligase Nedd $4 \mathrm{~L}$ targets activated Smad2/3 to limit TGFbeta signaling. Mol. Cell 36, 457-468 (2009).

37. Kretzschmar, M., Doody, J. \& Massague, J. Opposing BMP and EGF signalling pathways converge on the TGF-beta family mediator Smadl. Nature $\mathbf{3 8 9}$ 618-622 (1997).

38. Kim, J. E., Nakashima, K. \& de Crombrugghe, B. Transgenic mice expressing a ligand-inducible cre recombinase in osteoblasts and odontoblasts: a new tool to examine physiology and disease of postnatal bone and tooth. Am. J. Pathol. 165, 1875-1882 (2004).

39. Wang, Y. et al. Receptor subunit-specific action of oncostatin M in hepatic cells and its modulation by leukemia inhibitory factor. J. Biol. Chem. 275, 25273-25285 (2000).

40. Kubin, T. et al. Oncostatin $\mathrm{M}$ is a major mediator of cardiomyocyte dedifferentiation and remodeling. Cell Stem Cell 9, 420-432 (2011).

41. Lörchner, H. et al. Myocardial healing requires Reg3 $\beta$-dependent accumulation of macrophages in the ischemic heart. Nat. Med. 21, 353-362 (2015).
42. Iyer, S. V., Harpaz, R., LePendu, P., Bauer-Mehren, A. \& Shah, N. H. Mining clinical text for signals of adverse drug-drug interactions. J. Am. Med. Inform. Assoc. 21, 353-362 (2014)

43. West, N. R. et al. Oncostatin M drives intestinal inflammation and predicts response to tumor necrosis factor-neutralizing therapy in patients with inflammatory bowel disease. Nat. Med. 23, 579-589 (2017).

44. Frangogiannis, N. G. et al. Critical role of monocyte chemoattractant protein $1 /$ CC chemokine ligand 2 in the pathogenesis of ischemic cardiomyopathy. Circulation 115, 584-592 (2007).

45. van Rooijen, N. \& Sanders, A. Elimination, blocking, and activation of macrophages: three of a kind? J. Leukoc. Biol. 62, 702-709 (1997).

46. Nishimura, S. et al. IL-1alpha induces thrombopoiesis through megakaryocyte rupture in response to acute platelet needs. J. Cell Biol. 209, 453-466 (2015).

\section{Acknowledgements}

The authors are grateful to Minori Fukumoto and Noriko Yamanaka for their technical assistance. This study was supported by Grant-in-Aid for Scientific Research from the Japan Society for the Promotion of Science (JSPS, Japan) (to N.T. 16K09493, to N.T. H.S 18H02360, 18H04122, to H.A. 17K15990, to M.A. 16K10900), Grant-in-Aid for Scientific Research on Innovative Areas (to N.T. 26111003) and JST PRESTO (to N.T. JPMJPR 14M4). This research was also supported by the Japan Society for the Promotion of Science (JSPS) through the Funding Program for World-Leading Innovative R\&D on Science and Technology (FIRST Program) initiated by the Council for Science and Technology Policy (CSTP). K.O. was supported by British Heart Foundation (RG/16/15/ 32294). H.S. was supported by The Uehara Memorial Foundation.

\section{Author contributions}

H.A., N.T. and T.I. designed the study and were involved in all aspects of the experiments with technical help from M.A., M.L.N., J.W.-H., C.S. and K.O. and wrote the manuscript. S.N. performed hypoxia imaging analysis. M.S.M. performed RNA-seq analysis. K.K. and Y.N. were involved in the flow cytometry analysis. K.S., M.W., M.K. and T.S. were involved in in vivo studies. H.S., Y.H., T.Y. and I.M. designed the study and were involved in the histological studies. Y.N., M.K. and R.I. measured oxygen consumption of the cardiomyocytes. T.I., T.Y. and K.M. performed reporter analysis. R.N. and I.K. designed the studies and supervised overall project.

\section{Additional information}

Supplementary Information accompanies this paper at https://doi.org/10.1038/s41467 019-10859-w.

Competing interests: N.T. has received grant supports from DAIICHI SANKYO COMPANY. LIMITED, Bayer Yakuhin, Ltd and Astra Zeneca K.K. The remaining authors declare no competing interests.

Reprints and permission information is available online at http://npg.nature.com/ reprintsandpermissions/

Peer review information: Nature Communications thanks Merry Lindsey and other anonymous reviewer(s) for their contribution to the peer review of this work.

Publisher's note: Springer Nature remains neutral with regard to jurisdictional claims in published maps and institutional affiliations.

Open Access This article is licensed under a Creative Commons Attribution 4.0 International License, which permits use, sharing, adaptation, distribution and reproduction in any medium or format, as long as you give appropriate credit to the original author(s) and the source, provide a link to the Creative Commons license, and indicate if changes were made. The images or other third party material in this article are included in the article's Creative Commons license, unless indicated otherwise in a credit line to the material. If material is not included in the article's Creative Commons license and your intended use is not permitted by statutory regulation or exceeds the permitted use, you will need to obtain permission directly from the copyright holder. To view a copy of this license, visit http://creativecommons.org/ licenses/by/4.0/

(C) The Author(s) 2019 